\title{
ESTRUCTURA DE CAPITAL PARA EL CRECIMIENTO CORPORATIVO: CASO EL HOLDING ANDINO
}

\author{
CAPITAL STRUCTURE FOR CORPORATE GROWING: CASE HOLDING THE ANDES \\ Julio Alberto Hennings Otoya * \\ Docente Asociado de la Facultad de Ciencias Contables \\ Universidad Nacional Mayor de San Marcos-UNMSM / Lima-Perú \\ [Recepción: Marzo de 2014/ Conformidad: Mayo 2014]
}

\section{RESUMEN}

El presente artículo describe, analiza y explica la problemática de una corporación nacional en crecimiento, Andino Investment Holding (AIH), en su búsqueda por concretar una estructura de capital eficiente que posibilite su sostenibilidad de empresa en marcha, y que le permita profundizar el desarrollo conseguido en las últimas décadas. La investigación se basó en la revisión y análisis documental de la información económico financiera de la empresa que obra en la Superintendencia del Mercado de Valores, la Bolsa de Valores de Lima e informes de los evaluadores de riesgo. Igualmente, se contrastó y reforzó los hallazgos con entrevistas a funcionarios vinculados al caso. Finalmente, al observar la dirección del holding, la inviabilidad de seguir creciendo basándose en deuda, se decide abrir el capital a inversionistas externos, vía emisión y colocación en la Bolsa de Valores de Lima. Previamente, AIH y subsidiarias, se ven obligados a realizar los cambios organizacionales y de gobierno, acordes a la nueva condición, lo cual fortalecerá su desarrollo. Resultante de esta nueva estrategia de capital amparado en el mercado de capitales, el Holding disminuye sus costos financieros $\mathrm{y}$ obtiene el refuerzo patrimonial para consolidar su expansión y sostenibilidad económica.

\section{Palabras Clave:}

Estructura de capital; apalancamiento financiero; holding de empresas.

\section{ABSTRACT}

This article describes, analyzes and explains the problems of a national corporation growing Andino Investment Holding (AIH), in their quest to realize an efficient capital structure that enables its sustainability going concern, and that allows the further development achieved in the past decades. The research was based on the review and documentary analysis of economic and financial information company that works in the Superintendence of Securities Market, the Lima Stock Exchange and reports of risk assessors. Also was tested and reinforced the findings of interviews with officials linked to the case. Finally, observing the direction of the holding, the impossibility of continued growth based on debt, decides to open up capital to outside investors, by the issuance and placement on the Lima Stock Exchange. Previously, AIH and subsidiaries, are forced to make organizational and governance, in line with the new status changes, which will strengthen its development. Resulting from this new capital strategy covered in the capital market, the Holding decreases financial costs and obtains the patrimonial reinforcement consolidate its expansion and economic sustainability.

\section{Keywords:}

Capital structure; financial leverage; holding company.

* Doctor en Administración, Magister en Negocios Internacionales y Contador Público - UNMSM. Email: jhennings@speedy.com.pe 


\section{INTRODUCCIÓN}

A partir del año 2001 se observa un crecimiento constante del comercio exterior peruano, acompañado con un favorable superávit comercial que contribuye al incremento de reservas internacionales del país, mayor generación de empleo y una positiva sensación de optimismo empresarial. Es en esta realidad, en la cual si el Perú creció a un ritmo promedio de $5.9 \%$ en la década, las exportaciones e importaciones lo hicieron a una tasa de $20.5 \%{ }^{1}$.

Con estos antecedentes y expectativas por parte de los agentes privados, reforzados por estimaciones del sector público oficial del país, hace prever que el comportamiento dinámico del sector externo se va a mantener, impulsado de un lado por una economía interna creciente que demandaría un mayor volumen de importaciones, y por otro lado, mayores exportaciones provenientes de diferentes sectores, entre ellos el agrícola, construcción y minería, en las que se esperan inversiones por varias decenas de millones de dólares. Este crecimiento beneficiaría de manera directa a numerosas empresas locales ligadas al comercio y la logística internacional, que vienen planificando su crecimiento, como ocurre con el grupo de empresas que lidera Andino Investment Holding - AIH, cuyas actividades se relacionan con la provisión de servicios marítimos, logísticos e infraestructura para los intercambios de exportación e importación, dentro de los negocios internacionales.

\section{Gráfico $\mathrm{N}^{\circ} 1$}

Esquema de Actividades y Empresas del Grupo AIH

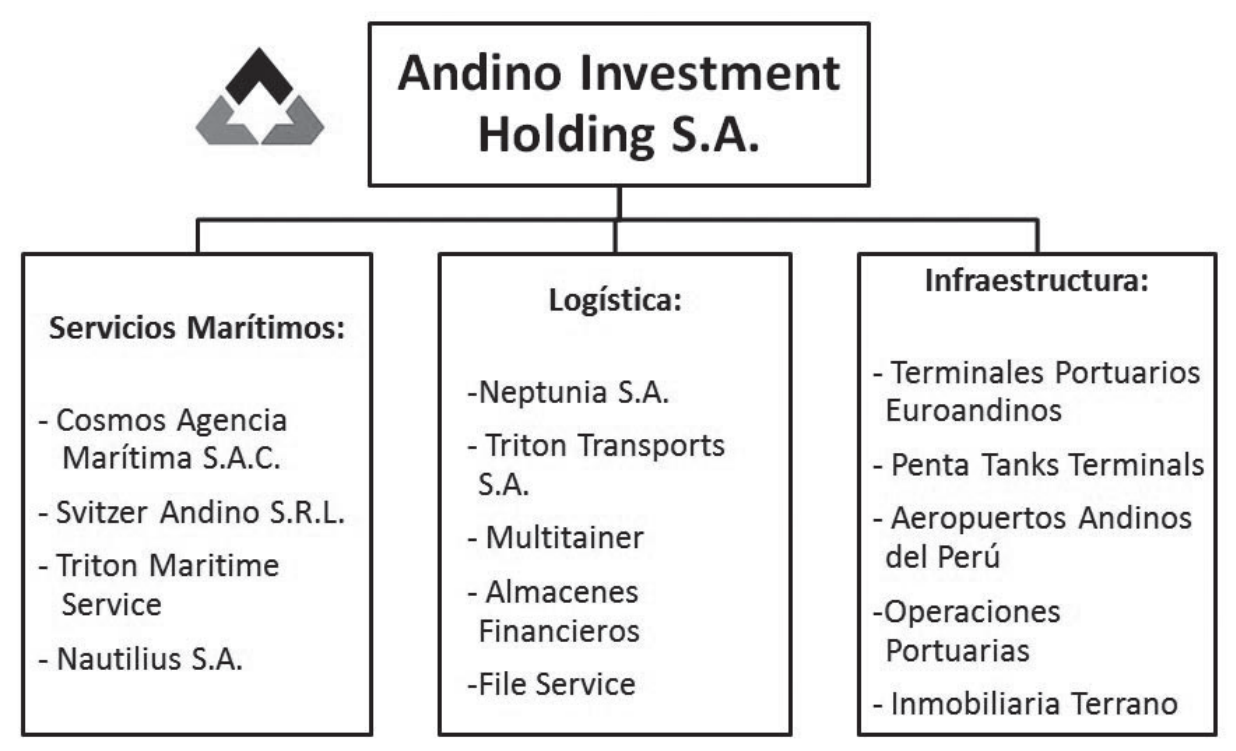

Fuente: Seminario SAB / Departamento de Análisis Financiero.

Justamente, para el desarrollo de la temática del presente estudio se escogió el caso del Holding Andino (AIH), el cual es un grupo corporativo de origen peruano que agrupa a 15 empresas que se desenvuelven en el rubro del comercio exterior, específicamente en tres líneas principales: Infraestructura, Servicios Marítimos y Servicios Logísticos (Gráfico $\mathrm{N}^{\circ} 1$ ). Las consideraciones para su elección fueron: su condición de grupo empresarial peruano en pleno crecimiento, su gestión profesional y las buenas perspectivas que ofrece el sector en que se desenvuelve.

\section{FUNDAMENTACIÓN DEL PROBLEMA}

Las perspectivas económicas empresariales del Holding Andino son positivas. El crecimiento futuro de este holding se sustenta en una combinación de (1) la expansión de las operaciones de sus empresas

$1 \mathrm{http}: / /$ www1.bcrp.gob.pe/consulta.asp?sIdioma=1\&sTipo=1\&sChkCount=35\&sFrecuencia=A

42/ QUIPURAMAVOC | Vol. 22(41) 2014 
establecidas, (2) sus concesiones y, (3) el desarrollo de nuevos proyectos.

Sobre la actuación del Grupo AIH, se puede resumir que al proporcionar una amplia gama de servicios portuarios y logísticos, ofreciéndoles a sus clientes los productos y servicios de todas sus unidades de negocios, los ha colocado en una posición de ventaja frente a sus competidores, que en su mayoría solo pueden ofrecer una parte de los varios servicios complementarios (agenciamiento marítimo, consolidación de carga, transporte, almacenamiento, entre otros), que los usuarios del comercio exterior requieren. Esta posibilidad de ofrecer servicios integrados, es el resultado favorable de un plan de desarrollo empresarial del Grupo Andino, que ha venido en el tiempo, expandiendo sus líneas de servicios a través de una estrategia de círculos concéntricos, consolidando cada unidad de negocio y luego haciendo que esta crezca, ampliando en cada caso el ámbito de los servicios de sus filiales.

De igual forma, el crecimiento previsto para las exportaciones e importaciones en los siguientes años, beneficiará de manera directa las actividades de Neptunia, Cosmos y Tritón Transports. En la actualidad, las empresas más antiguas del Grupo Andino, así como, el puerto concesionado de Paita, los Aeropuertos del Sur y, en general, el resto de empresas de servicios, se verán favorecidas con un mayor movimiento comercial y volúmenes de carga.

Dado que la tenencia de grandes áreas es crucial en el negocio que el Grupo actúa, en la última década han ido adquiriendo terrenos y desarrollando proyectos y negocios en los mismos. En la actualidad, cuenta con más de 1.2 millones de metros cuadrados de terrenos en zonas claves de Lima y provincias, cuyo valor comercial supera los 200 millones de dólares, lo que da al grupo empresarial una base de valor muy sólida. De ellos, aproximadamente $750,000 \mathrm{~m}^{2}$ están libres y serán utilizados para desarrollar proyectos de negocios, lo cual cimienta su futuro crecimiento. El terreno de mayor extensión $\left(600,000 \mathrm{~m}^{2}\right)$, está ubicado en Ventanilla, a $14 \mathrm{~km}$ al norte del Puerto del Callao, en el cual el Holding Andino ha previsto construir un puerto de gráneles limpios, el cual también podría incluir concentrados de mineral a futuro.
Respecto a las mejoras organizacionales, AIH ha venido implementando una serie de medidas para consolidar su estructura administrativa y organizativa. Creada en el año 2005, su objetivo es coordinar la estrategia del Grupo hacia adelante, supervisar a las empresas operativas y representar al grupo ante las autoridades y reguladores. En el 2010, se creó Andino Servicios Corporativos, la unidad de servicios del grupo, con el propósito de centralizar los servicios de back office de todas las empresas del Grupo, y así obtener sinergias por un uso más eficiente de los recursos. Adicionalmente, el Grupo Andino continúa con el proceso de reestructuración societaria con el fin de aumentar las eficiencias de las subsidiarias del holding.

Sustentado el origen que augura perspectivas favorables de crecimiento de la organización, se debe resaltar que el año 2011 marcará en la historia del Grupo Andino, su punto de quiebre en su discurrir financiero local. Así, el 15 de febrero de 2011, AIH recurre a financiamiento del exterior, tomando un préstamo sindicado por US\$ 85 millones, transacción que fuera liderada por Goldman Sachs Credit Partners L.P. de New York, importante subsidiaria del Banco de Inversión Goldman Sachs de Estados Unidos. Esta operación, es solicitada por el grupo mayoritario que conformaba el Holding Andino para viabilizar la compra de participaciones minoritarias en las empresas del grupo (fundamentalmente de posesión del grupo empresarial chileno Von Appen), pero deja al holding con un nivel de endeudamiento muy alto, que a setiembre de ese mismo año 2011 alcanza a 2.83 veces su patrimonio (ver Tabla $\mathrm{N}^{\circ} 1$ ).

Es en ese momento, que se acuerda restablecer un nivel de endeudamiento razonable para la organización, considerando en su lineamiento financiero, la necesidad de captar fondos para pre-pagar la deuda anterior, además de cubrir los compromisos pactados previamente por el Grupo Andino, como el aporte de 20 millones de dólares a los proyectos de Terminales Portuarios Euroandinos (TPE) en la concesión del Puerto de Paita, donde participa conjuntamente con la firma portuguesa Mota Engil.

Conviene agregar, que como consecuencia al alto nivel de deuda alcanzado por el Holding a partir del 
año 2011, su rendimiento -ver ratios de solvencia y rentabilidad de la tabla $\mathrm{N}^{\circ} 1$ - cae en forma pronunciada, afectándose por los costos financieros (de un rendimiento sobre patrimonio en el año 2009 de
6.7\% y en el año 2010 de 5.3\%, baja a $1.4 \%$ en el año 2011 , con tendencia a empeorar si no hay cambios de rumbo).

Tabla $\mathbf{N}^{\circ} 1$

Indicadores Financieros del Holding Andino - AIH

(Del 2009 a Set. 2011)

\begin{tabular}{|c|c|c|c|c|}
\hline & 30.09 .11 & 30.09 .10 & 2010 & 2009 \\
\hline \multicolumn{5}{|l|}{ Ratios de Liquidez } \\
\hline Razón Corriente & $1.31 \%$ & $0.64 \%$ & $0.91 \%$ & $0.94 \%$ \\
\hline Prueba Ácida & $1.02 \%$ & $0.69 \%$ & $0.79 \%$ & $0.82 \%$ \\
\hline \multicolumn{5}{|l|}{ Ratios de Gestión } \\
\hline Margen Operativo & $8.3 \%$ & $5.7 \%$ & $5.9 \%$ & $6.9 \%$ \\
\hline \multicolumn{5}{|l|}{ Ratios de Solvencia } \\
\hline Endeudamiento Patrimonial & $2.83 \%$ & $0.91 \%$ & $0.88 \%$ & $0.94 \%$ \\
\hline Cobertura de Interéses & $0.75 \%$ & $0.43 \%$ & $2.74 \%$ & $3.39 \%$ \\
\hline \multicolumn{5}{|l|}{ Ratios de Rentabilidad } \\
\hline Rentabilidad neta sobre Patrimonio & $1.3 \%$ & $3.9 \%$ & $4.7 \%$ & $6.4 \%$ \\
\hline Rentabilidad neta sobre Ventas & $0.3 \%$ & $2.1 \%$ & $2.5 \%$ & $3.3 \%$ \\
\hline Utilidad neta sobre Ventas & 0.021 & 0.106 & 0.128 & 0.165 \\
\hline Dividendos por Acción & 0.018 & 0.011 & 0.024 & 0.047 \\
\hline
\end{tabular}

Fuente: AIH.

\section{MARCO DE REFERENCIA}

Tomando las palabras del Dr. Mauro Guillén (2012), profesor de la Escuela de Warthon: "Normalmente, los países suelen primero atraer inversión del resto del mundo y, con el tiempo y su crecimiento, las empresas locales comienzan a acumular destrezas, recursos o capacidades con potencial para ser explotados (incluso) en otros países". Se puede afirmar, que esto lo observamos en el grupo de $\mathrm{AIH}$, donde sus destrezas y capacidades, surgidas al calor de la apertura comercial del Perú y beneficiadas con la desregulación del mercado que monopolizaba las actividades del comercio exterior, de las reformas financieras y privatizaciones, han transformado los servicios brindados a exportadores e importadores, y posibilitado el desarrollo empresarial en un sector donde hasta hace una generación, se encontraba sin empresas de envergadura.

En cuanto al marco teórico financiero, se observa que las decisiones de financiamiento tomadas por el grupo empresarial Andino Investment Holding, para obtener capital de trabajo y posibilitar su crecimiento -incluyendo necesidades de inversión-, guardan concordancia con Fernández, De Rojas y David (2004), cuando afirman que: "la financiación interna no cubre las necesidades de inversión de nuestras empresas(de Iberoamérica), razón por la cual deben acudir a la financiación externa, prefiriendo la deuda a la emisión de acciones". La referencia aludida por estos expertos es a la teoría del "pecking order" o de la jerarquización financiera.

De igual manera, en línea con la teoría de la jerarquización financiera, sus autores Myers y Majluf (1984), sostienen que debido a problemas de selección adversa las empresas prefieren deuda a acciones, debido a los más bajos costes de información asociados con emisión de deuda. Justamente, las decisiones de financiamiento del Grupo Andino siguió el orden antes mencionado en sus primeras etapas de desarrollo. 
Sin embargo, se debe considerar que el orden en que las empresas buscan agenciarse recursos financieros para sus operaciones: primero financiamiento interno, segundo deuda y finalmente, emisión de acciones, reviste una lógica fácil de entender en las decisiones financieras de muchas empresas, pero las excepciones, vinculadas a la preferencia de ampliar capital (emitir nuevas acciones) antes de mayor deuda, se da en la eventualidad que tal orden dificulte su viabilidad económica en el tiempo, un ejemplo, cuando los volúmenes de deuda acumulados frente al patrimonio de la empresa, ponen en riesgo la eficiencia y sostenibilidad de la organización. También podrá "el pecking order" natural alterarse, cuando el que toma la decisión de contraer deuda o ampliar capital para sostener las operaciones o ejecutar nue- vos proyectos resulta "averso"al riesgo, primando en este caso, la tendencia conductual del directivo de preferir menor riesgo, aunque su decisión resulte más onerosa.

\section{INTERPRETACIÓN DE RESULTADOS}

En el estudio de caso, se observa documentalmente y a través del análisis de los Estados Financieros, el momento crítico del Grupo investigado AIH al facturar a setiembre 2010 un total de S/.320 millones y avanzar a setiembre 2011 con S/. 363 millones. Sin embargo, contrariamente la utilidad en dichos periodos se retrae, así en el mismo periodo 2010 de S/. 10.5, baja en el año siguiente 2011 a S/. 2.1 millones de soles (ver tabla $\mathrm{N}^{\circ} 2$ ).

\section{Tabla $\mathbf{N}^{\circ} 2$}

Ingresos del Grupo Andino - AIH

(Del 2009 a Set. 2011)

\begin{tabular}{|c|c|c|c|c|}
\hline & 30.09 .11 & 30.09 .10 & 2010 & 2009 \\
\hline & \multicolumn{4}{|c|}{ En miles de S/. } \\
\hline Total de Ingresos Operacionales & 362,542 & 319,899 & 433,915 & 391,016 \\
\hline Costo de Ventas & - & - & - & - \\
\hline Utilidad Bruta & 362,542 & 319,899 & 433,915 & 391,016 \\
\hline Utilidad Operativa & 30,067 & 18,378 & 25,449 & 27,137 \\
\hline Utilidad (Pérdida) Neta del Ejercicio & 1,112 & 10,544 & 12,708 & 16,448 \\
\hline
\end{tabular}

Fuente: AIH.

La paradoja "ingresos crecientes frente a utilidades decrecientes" se explica por el incremento sustancial de los costos financieros, resaltando los generados a partir de febrero 2011, al haber contraído un crédito sindicado liderado por Goldman Sachs por US\$ 85 millones, para comprar participaciones minoritarias, capitalizar algunos proyectos y repagar deuda local, lo que eleva su nivel de endeudamiento a 2.83 veces del patrimonio en set. 2011. Este hecho, revistió un alto riesgo para la sostenibilidad financiera y la continuidad de negocio de la organización empresarial.

La situación antes descrita, se logra superar cambiando su estrategia financiera, es decir, incremento de patrimonio emitiendo y ofertando nuevas acciones en el mercado de capitales peruano, consiguiendo finalmente el 2 de febrero de 2012, la venta del 99.4\% de las acciones emitidas para oferta pública (IPO) en la Bolsa de Valores de Lima, obteniendo S/. 114.85 millones de soles de ingreso líquido, que le permitieron amortizar (pre-pago de la deuda cara) S/.33.75 millones al crédito sindicado de Goldman Sachs, invertir S/. 72.90 millones en la modernización del puerto concesionado de Paita, y el saldo de S/. 8.20 millones para capital de trabajo. La nueva estrategia de financiación directa a través del mercado de capitales peruano reforzó su patrimonio empresarial, permitiendo menores costos de capital y con ello, posibilitó continuar los planes de desarrollo del holding andino $(\mathrm{AIH})$. 


\section{CONCLUSIONES}

1. La estrategia de financiación directa seguida por el grupo empresarial Andino Investment Holding, utilizando la Bolsa de Valores de Lima para emitir y colocar nuevas acciones de capital, les permitió reforzar su patrimonio empresarial, permitiéndoles operar sus actividades e inversiones con menores costos de capital, y posibilitando así, continuar los planes de expansión y desarrollo sostenible del grupo corporativo peruano.

2. Las exigencias de los entes normativos, supervisores y, gestores del mercado de capital peruano, llámense la Superintendencia del Mercado de Valores y la Bolsa de Valores de Lima, obligan mediante requisitos a las empresas que quieren acceder al mercado financiero como emisores de títulos de capital (ejemplo el caso del Grupo Andino con acciones) o como emisores de deuda (bonos, papeles comerciales), a ordenar su manejo empresarial y tecnificar sus decisiones a través de la implementación de un "gobierno corporativo", aspecto que resultan de vital conveniencia para el desarrollo futuro de las organizaciones empresariales.

3. El orden en que las empresas buscan agenciarse recursos financieros según la teoría del pecking order: primero financiamiento interno, segundo deuda y finalmente, emisión de acciones (participación de capital), reviste una lógica fundamentada fácil de entender y aceptada en las decisiones financieras de muchas empresas, pero las excepciones, vinculadas a la preferencia de ampliar capital (emitir nuevas acciones) antes de optar por mayor deuda, se da ante la eventualidad que tal orden de priorización financiera, dificulte su viabilidad económica, un ejemplo podría darse, cuando los volúmenes de deuda acumulados frente al patrimonio de la institución, pongan en riesgo la eficiencia y sustentabilidad de la organización, como fue el caso de Grupo Andino.

\section{REFERENCIAS BIBLIOGRÁFICAS}

1. Bodie Z.y Merton R. (2003). Finanzas. Pearson Educación. México.

2. Fernández M., De Rojas M. y David G. (2004). Contrastación de la Teoría del Pecking Order. Universidad de Valladolid. España.

3. Guillen M. y Garcia E. (2012). Emerging Marketing Rule. Growth Strategies of the New Global Giants. McGraw-Hill Professional. EE.UU.

4. Myers, S. y Majluf, N. (1984). Corporate Financing and Investment Decisions when firms have information that investors do not have. NBER Working Paper No. 1396. Journal of Financial Economics, Vol. 13, No. 2, 1984, pp. 187-221. EE.UU.

5. Myers, S. (2001). Capital Structure. The Journal of Economics Perspectives. Vol. 15. $N^{\circ} 2$. Págs. 81-102. United States of America.

6. Mongrut, S. Fuenzalida, D. Pezo, G. y Teply, Z. (2010). "Explorando Teorías de Estructura de Capital en Latinoamérica", dirigido por la Universidad Técnica Federico Santa María, Valparaíso, Chile.

7. Vargas Loret de Mola Carlos. Entrevistas (2) publicadas en la Revista Empresarial "G" de Gestión. $\mathrm{N}^{\circ} 14$ de enero 2012 y $\mathrm{N}^{\circ} 15$ de febrero 2012. Edición Revista Amauta del Grupo El Comercio. Lima.

\section{REFERENCIAS ELECTRÓNICAS}

1. http://www.bvl.com.pe/inf_financiera71500 AIHC1.html

2. http://www1.bcrp.gob.pe/consulta.asp?sIdio$\mathrm{ma}=1 \& \mathrm{sTipo}=1 \& \mathrm{sChkCount}=35 \&$ sFrecuen $\mathrm{cia}=\mathrm{A}$

3. http://www.mef.gob.pe/contenidos/pol_econ/ marco_macro/MMM2014_2016_Rev.pdf

4. http://www.smv.gob.pe/ConsultasP8/temp/ xqmpj1la.y4h.pdf 